\title{
Prevalence of ocular inflammation among patients with active tuberculosis or nontuberculous mycobacterial infections in a tertiary hospital in Japan: a retrospective observational study
}

Hiromi Ohara

"Hiroshima Daigaku"

Yosuke Harada ( $\square$ yharada@hiroshima-u.ac.jp)

"Hiroshima Daigaku" https://orcid.org/0000-0001-9827-4963

Tomona Hiyama

"Hiroshima Daigaku"

Ken Yamane

Yamane Eye Clinic

Maria Higaki

Yoshijima Hospital

Takayuki Kobayashi

Yoshijima Hospital

Yasuhiko Ikegami

Yoshijima Hospital

Yuki Yuasa

"Hiroshima Daigaku"

Yoshiaki Kiuchi

"Hiroshima Daigaku"

Original research

Keywords: Ocular inflammation, Prevalence, Tuberculosis, Nontuberculous mycobacterial infection

Posted Date: May 26th, 2020

DOI: https://doi.org/10.21203/rs.3.rs-30472/v1

License: (c) (1) This work is licensed under a Creative Commons Attribution 4.0 International License.

Read Full License 


\section{Abstract}

Background This study aimed to elucidate the prevalence of ocular involvement among patients with active tuberculosis (TB) or nontuberculous mycobacterial (NTM) infection in a Japanese hospital.

Materials and Methods Patients with active TB or NTM infection at Yoshijima Hospital from April 2017 to July 2018 were included in this retrospective study. All patients underwent ophthalmic examinations, including fundus evaluation under pupil dilation, before initiation of antibiotic therapy. Patients with ocular inflammation were regularly followed up by ophthalmologists.

Results In total, 101 patients with active TB and 27 patients with active NTM infection underwent ophthalmic examinations during the study period. No ocular symptoms were reported. Seven patients with TB (6.9\%) had ocular inflammation; four had bilateral involvement. In these seven patients, ocular inflammation comprised retinal vasculitis $(n=2)$, anterior uveitis $(n=2)$, choroiditis $(n=2)$, and vitritis $(n=1)$. Female sex was associated with higher prevalence of ocular inflammation among patients with TB. Conversely, no patients with NTM infection had ocular inflammation.

Conclusion Ocular inflammation was present in approximately $7 \%$ of patients with active TB. Although TB choroiditis is presumed to be rare in Japan, approximately $30 \%$ of the patients with ocular inflammation exhibited choroidal lesions in this study. In contrast, no ocular inflammation was observed among patients with systemic NTM infection.

\section{Background}

The prevalence of tuberculosis (TB) in Japan has decreased since the introduction of the Tuberculosis Prevention Law in 1951. However, the number of patients with new-onset TB was 15,590 in $2018{ }^{1}{ }^{1}$ the prevalence was 12.3 per 100,000 people, which is several-fold higher than the prevalences of other developed countries (e.g., the United States $[2.8 / 100,000],{ }^{2}$ Canada $[4.9 / 100,000],{ }^{3}$ and Sweden $[5.3 / 100,000]) .{ }^{4}$ Thus, TB infection continues to require careful monitoring in Japan.

The severe ocular inflammation due to TB uveitis causes irreversible impairment of visual acuity. 5,6 There have been several reports of TB uveitis prevalence among causes of ocular inflammation in Japan.

Ohguro et $\mathrm{al}^{7}$ reported that the prevalence of TB uveitis was $1.4 \%$ among all types of ocular inflammation, while Goto et $\mathrm{al}^{8}$ reported that its prevalence was $0.7 \%$, indicating that the proportion of TB associated ocular inflammation in Japan is lower than that reported in India ${ }^{9,10}$ or Bangladesh. ${ }^{11}$ Suzuki et $\mathrm{al}^{12}$ found that periphlebitis was the most common manifestation of Japanese ocular TB, while choroiditis is reportedly the most common manifestation in other countries. ${ }^{13,14}$

Nontuberculous mycobacterial (NTM) infections have increased worldwide in recent decades ${ }^{20}$ However, epidemiologic characteristics of NTM remain largely unclear, because NTM infections are not required to be reported to public health authorities in most countries (unlike TB) ${ }^{21}$ In Japan, the prevalence of 
pulmonary NTM was reportedly 29.0 per 100,000 people, according to data collected from 2009 to $2014 .{ }^{22}$ NTM are also known as causative organisms involved in ocular infection. ${ }^{23}$ NTM ocular infections cause periocular, adnexal, ocular surface, and intraocular manifestations; they are often resistant to medical therapy. ${ }^{23}$ Most cases of NTM ocular infection involve keratitis that occurs after interventions such as cataract surgery, laser in situ keratomileusis, or penetrating keratoplasty. ${ }^{23}$ Notably, intraocular inflammation associated with systemic NTM infection is very rare. To the best of our knowledge, there have been few reports regarding intraocular infections due to systemic NTM infection; most cases involved immunocompromised patients with acquired immunodeficiency disease.

The accumulation of data from various countries/areas is of considerable importance, because the rate of ocular inflammation in patients with active TB and clinical characteristics of TB uveitis reportedly exhibit considerable variation worldwide. Real-world data are also needed regarding the prevalence of ocular inflammation in non-immunocompromised patients with systemic NTM infection. In the present study, we conducted dilated fundus examination to elucidate the prevalence and characteristics of ocular involvement among patients with active systemic TB or NTM infection in Yoshijima Hospital, a TBtreatment core hospital in Hiroshima, Japan.

\section{Materials And Methods}

This observational study included patients with active TB or NTM infection at Yoshijima Hospital from April 2017 to July 2018. The study adhered to the tenets of Declaration of Helsinki and was approved by the Institutional Review Board of Hiroshima University and Yoshijima Hospital. It was granted a waiver of informed consent by the Institutional Review Board of Hiroshima University and Yoshijima Hospital because the analysed data consisted of de-identified records.

Inclusion criteria were diagnosis of active systemic TB including pulmonary tuberculosis and miliary tuberculosis, or active systemic NTM infection. Data collected from the Department of Pulmonology included age, sex, ethnicity, date of diagnosis, medical history, family history, follow-up period, and images obtained (e.g., chest X-ray or computed tomography).

When patients were diagnosed with these diseases, they were referred to ophthalmologists prior to initiation of systemic antibiotics. Uveitis specialists (H.O, K.Y, M.H, and T.K) evaluated the patients by dilated fundus examination.

Standard therapy for systemic TB comprised four-drug therapy (isoniazid, rifampin, pyrazinamide, and ethambutol) for 2 months, followed by two-drug therapy (isoniazid, rifampin) for 4 months. Therapy for NTM infection depended on the species. Standard treatment for Mycobacterium avium complex (Mycobacterium intracellulare and Mycobacterium avium) comprised rifampin, clarithromycin, and ethambutol. For Mycobacterium kansasii, standard treatment comprised isoniazid, rifampin, and ethambutol. 
Ophthalmic examinations included decimal best-corrected visual acuity (BCVA) and intraocular pressure (IOP) measurement; assessments of scleritis, anterior chamber inflammation, vitreous haze, retinal vasculitis, and chorioretinal lesions were also determined, based on the medical record. If a patient exhibited ocular inflammation, fundus photographs (Kowa Vx-10/Vx-20a, Kowa Company. Ltd., Tokyo, Japan) and slit lamp photographs were collected. When anterior chamber cells, vitreous haze, or scleritis were observed, inflammation was evaluated in accordance with the Standardization of Uveitis Nomenclature guidelines and scleritis grading system. ${ }^{24}$ When possible, all patients with ocular inflammation were followed up after antibiotic therapy.

Univariate analysis, multivariate analysis, Pearson's correlation coefficient, and t-tests were used to analyse risk factors for tuberculous uveitis. JMP software, version 11 (SAS Inc, Cary, NC, USA), was used for statistical analyses, and $P<0.05$ was considered to be statistically significant.

\section{Results}

In total, 101 patients with active TB and 27 patients with active NTM infection underwent ophthalmic examination in this study (Tables 1 and 2). Among the patients with TB, the mean age at examination was $68.9 \pm 20.9$ years (range, $19-100$ years) and $57.4 \%$ of these patients were men. Ninety-three patients (92.1\%) were Japanese. The most common form of disease was pulmonary TB ( $n=89,88.1 \%)$.

Extrapulmonary TB (e.g., tuberculous pleurisy, bronchial tuberculosis, tuberculous lymphadenitis, and/or intestinal tuberculosis) was observed in one-sixth of patients with pulmonary TB. Miliary tuberculosis (5.0\%) and tuberculous pleurisy without pulmonary TB (5.9\%) were also observed among patients in this study. Twenty-five patients $(24.8 \%)$ had reactivated tuberculosis, according to their medical records.

Ophthalmic examinations of patients with TB revealed that decimal BCVAs were as follows: $<0.1$ in nine eyes, $0.1-0.3$ in 15 eyes, $0.4-0.6$ in 36 eyes, $0.7-0.9$ in 41 eyes, and $\geq 1.0$ in 101 eyes. Patients with low BCVA ( $\leq 0.3)$ had cataract (15 eyes), age-related macular degeneration (four eyes), glaucoma (four eyes), ocular trauma (one eye), and epiretinal membrane (one eye). IOP was within the normal range for all patients, although 18 eyes were undergoing treatment with anti-glaucoma eye drops. While 30 patients (51 eyes) had ocular disease, none had a history of previous ocular inflammation (Table 1). Despite the lack of ocular symptoms, seven of the 101 patients with TB (6.9\%) had ocular inflammation (Tables 1 and 3). Six of these seven patients (85.7\%) were women; six were Japanese and one was Filipino. Four of these seven patients had bilateral involvement; two of the seven patients had retinal vasculitis, two had anterior uveitis, two had chorioretinitis, and one had vitritis. All patients with ocular inflammation exhibited active disease. While three patients were lost to follow-up, four patients were followed up until the resolution of inflammation. Of these four patients, topical betamethasone in combination with antituberculosis therapy (ATT) was administered to one patient with anterior uveitis, while photocoagulation of the nonperfusion area was performed in one patient with retinal vasculitis; in the other two patients, ocular inflammation was resolved solely with ATT. The mean duration until resolution of ocular inflammation was $42.5 \pm 23.7$ days (range, 29-77 days). The presence of extrapulmonary TB was not associated with the prevalence of ocular inflammation $(p=0.47)$. Univariate analysis revealed 
that female sex $(p=0.01)$ and hematological diseases $(p=0.02)$ were associated with ocular involvement (Table 1). No patients with immunosuppressant therapy exhibited ocular inflammation. Multivariate analysis revealed that female sex was significantly associated with ocular involvement $(p=0.04)$ (Table 4).

Among the patients with NTM infection, the mean age at examination was $67.7 \pm 8.8$ years (range, 5181 years) and $29.6 \%$ of these patients were men. All patients had been diagnosed with atypical mycobacterial pneumonia. The most common species was $M$. intracellulare $(63.0 \%)$, followed by $M$. avium (44.4\%), M. kansasii (3.7\%), Mycobacterium shimoidei (3.7\%), and Mycobacterium marseillense (3.7\%). A patient infected with M. shimoidei and $M$. marseillense also exhibited coinfection with $M$. intracellulare. Ophthalmic examinations of patients with NTM infection revealed that decimal BCVAs were as follows: $<0.1$ in one eye, $0.1-0.3$ in three eyes, $0.4-0.6$ in four eyes, $0.7-0.9$ in 11 eyes, and $\geq 1.0$ in 35 eyes. Patients with low BCVA ( $\leq 0.3$ ) had cataract (two eyes), glaucoma (one eye), and myopic choroidal neovascularisation (one eye). IOP was within the normal range for all patients, although six eyes were undergoing treatment with anti-glaucoma eye drops. While 10 eyes (six patients) had ocular disease, none had a history of previous ocular inflammation (Table 2). In contrast to the findings in patients with TB, there were no signs of ocular inflammation among patients with NTM infection.

\section{Discussion}

The prevalence of TB infection is higher in Japan than in other developed countries. ${ }^{25}$ In Japan, ophthalmologists are asked to perform ocular evaluation of patients with TB or NTM infection prior to treatment, especially before initiation of ethambutol therapy. However, there have been no reports regarding the prevalence of ocular inflammatory disease (based on dilated fundus examination) in Japanese patients with active TB or NTM infection. Here, we found that $6.9 \%$ of patients with active TB had ocular inflammation. Conversely, we found no ocular inflammation in patients with active NTM infection. Among the seven patients with TB who exhibited ocular involvement, two had chorioretinitis, which was previously reported to be a rare type of ocular inflammation among Japanese patients with TB. ${ }^{12}$ In all patients for whom follow-up data were available, ocular inflammation resolved with ATT alone (i.e., without systemic corticosteroid therapy). Our results indicated that TB-associated choroiditis among Japanese patients might be more common than previously reported, as it might be masked due to a lack of symptoms or possible spontaneous improvement with ATT.

The prevalence of ocular involvement in patients with systemic TB varies widely among reports. ${ }^{15-19}$ Donahue $^{15}$ reported a prevalence of 154 patients among 10,524 total patients with TB (1.4\%) in a TB sanatorium in the United States; Biswas et al ${ }^{16}$ reported a prevalence of $1.39 \%$ among 1,005 Indian patients. Higher prevalence in a tertiary hospital was reported in the Philippines (6.8\%). ${ }^{17}$ Beare et al ${ }^{18}$ observed a prevalence of $2.8 \%$ among patients with TB in Malawi $(94 \%$ of the patients exhibited concomitant human immunodeficiency virus [HIV] infection). A study from Spain revealed that $18 \%$ of patients with systemic TB had ocular involvement (45\% of the patients with TB were HIV-positive).$^{19}$ The 
prevalence of ocular inflammation in the present study was much higher than the prevalences of previous reports from the United States or India, ${ }^{15,16}$ and nearly identical to the prevalence observed in the Philippines. ${ }^{17}$ The elevated prevalence of ocular inflammation in the present study may be related to higher mean age of patients included in this study, compared with the ages of patients in the previous reports. Intraocular TB is presumed to result from direct ocular infection. Previous studies have found evidence of direct infection by Mycobacterium tuberculosis in retinal pigment epithelium, whereby $M$. tuberculosis is phagocytosed by retinal pigment epithelium and replicates in retinal pigment epithelium cells; these findings suggested that the retinal pigment epithelium serves as a reservoir of $M$. tuberculosis. ${ }^{26,27}$ Accordingly, ocular TB is presumed to occur due to reactivation of $M$. tuberculosis that was transmitted to the eye through the bloodstream from other tissues (mostly lung) and survived in ocular tissue (especially in retinal pigment epithelium). Theoretically, longer bacterial survival in tissue may increase the likelihood of bacterial activation. The immune response decreases with advancing age, such that $M$. tuberculosis in ocular tissue may be activated and lead to higher ocular involvement. ${ }^{28-30}$

Patients with ocular inflammation were predominantly women in this study; this finding is consistent with the results of previous epidemiological reports showing that female sex is a risk factor for concurrent extra-pulmonary TB. ${ }^{31,32}$ The causes of sex-related differences in the occurrence of TB are not well understood. Dissimilarities regarding the immune system, healthcare access, or socioeconomic factors between men and women have been suggested to influence these differences. ${ }^{33}$ Martinez et al ${ }^{34}$ reported that differences in TB rates between sexes may be due to differences in transmission dynamics. Further studies are needed to understand the exact mechanism underlying the sex-related difference in occurrence of extrapulmonary TB.

While the present study revealed a high prevalence of ocular inflammation among patients with systemic TB, no instances of intraocular involvement were observed among patients with systemic NTM infection. Despite the small sample size in present study, the results were consistent with the prior observations that NTM infection was less frequently involved in ocular inflammation. In a systematic review, Kheir et $\mathrm{al}^{23}$ revealed that uveitis was very rare $(2.2 \%)$, while the most common type of ocular inflammation comprised ocular surface infection (74.0\%); additionally, more than half of the patients with NTM-related uveitis were immunocompromised due to HIV/acquired immunodeficiency disease.

There were several limitations in this study. First, the sample size was small, and additional patients are needed to validate our findings. We collected these data by including consecutive patients with active systemic TB, since the beginning of this project. Continuous surveys will confirm our findings regarding the prevalence of ocular involvement among Japanese patients with systemic TB. Notably, the geographical distribution of TB also varies within Japan, such that the incidence of new TB cases can reach 6-20.5 per 100,000 people in some prefectures. In Hiroshima, the incidence of new TB cases was reported to be 10.3 per 100,000 , which was slightly lower than the overall incidence in Japan. ${ }^{1}$ Similar studies are needed in each prefecture to more precisely assess the prevalence of ocular inflammation among Japanese patients with active TB. Second, precise medical records could not obtained for some 
patients, because they had extremely long histories of TB or NTM infection. Finally, $40 \%$ of patients with ocular inflammation were lost to follow-up after ATT. More patients with ocular inflammation that is monitored by ophthalmologists are needed to confirm whether the ocular inflammation associated with systemic TB can be resolved by ATT.

\section{Conclusions}

In conclusion, we observed a higher prevalence of ocular inflammation among Japanese patients with active systemic TB, nearly identical to the prevalence reported in the Philippines. Female sex was associated with ocular inflammation among patients with active TB. Moreover, ocular inflammation responded to ATT without systemic corticosteroid. Ocular inflammation involving the choroid was more common than expected, based on previous reports. Notably, ocular inflammation was not observed among patients with systemic NTM infection. This study provides important real-world data regarding the prevalence and characteristics of ocular involvement in Japanese patients with active TB or NTM infection.

\section{List Of Abbreviations}

TB: tuberculosis

NTM: nontuberculous mycobacterial

BCVA: best-corrected visual acuity

IOP: intraocular pressure

ATT: antituberculosis therapy

HIV: human immunodeficiency virus

\section{Declarations}

\section{Ethics approval and consent to participate}

This study was approved with a waiver of informed consent by the ethics committee of Hiroshima University and Yoshijima Hospital (Approved number: 1937).

\section{Consent for publication}

Not applicable.

\section{Availability of data and materials}


The datasets generated and analyzed during the current study are available from the corresponding author on reasonable request.

\section{Competing interests}

The authors declare that they have no competing interests.

\section{Funding}

This research did not receive any specific grant from funding agencies in the public, commercial, or notfor-profit sectors.

\section{Authors' contributions}

$\mathrm{YH}$ and $\mathrm{KY}$ conceived the study design. $\mathrm{HO}, \mathrm{MH}, \mathrm{KY}$, and $\mathrm{YI}$ collected data. $\mathrm{HO}, \mathrm{TH}$, and $\mathrm{YH}$ wrote the article. $\mathrm{OH}$ and $\mathrm{YY}$ performed statistical analyses. $\mathrm{YK}$ performed critical revision of the manuscript.

\section{Acknowledgements}

We thank Ryan Chastain-Gross, Ph.D., from Edanz Group (https://en-author-services.edanzgroup.com) for editing a draft of this manuscript.

\section{References}

1. Department of Epidemiology and Clinical Research, the Research Institute of Tuberculosis: Tokyo, Japan. Tuberculosis in Japan - annual report 2017 (2018) Tuberculosis Surveillance Center. http://www.jata.or.jp/rit/ekigaku/en/statistics-of-tb/ Accessed 1 March 2020

2. TB Incidence in the United States, 1953-2018 (2019) Centers for Disease Control and Prevention. https://www.cdc.gov/tb/statistics/tbcases.htm Accessed 1 March 2020

3. Tuberculosis: M (2019) Government of Canada. https://www.canada.ca/en/publichealth/services/diseases/tuberculosis/surveillance.html Accessed 1 March 2020

4. Tuberculosis control in Sweden (2018) Karolinska Institutet. https://openarchive.ki.se/xmlui/bitstream/handle/10616/46472/Thesis_Jerker_Jonsson.pdf? sequence=1\&isAllowed=y Accessed 1 March 2020

5. Malalis JF, Goldstein DA (2015) Advances in tuberculosis-associated uveitis. Int Ophthalmol Clin 55:37-46

6. Patel SS, Saraiya NV, Tessler HH, Goldstein DA (2013) Mycobacterial ocular inflammation: delay in diagnosis and other factors impacting morbidity. JAMA Ophthalmol 131:752-758

7. Ohguro N, Sonoda K-H, Takeuchi M et al (2012) The 2009 prospective multi-center epidemiologic survey of uveitis in Japan. Jpn J Ophthalmol 56:432-435

8. Goto H, Mochizuki M, Yamaki K et al (2007) Epidemiological survey of intraocular inflammation in Japan. Jpn J Ophthalmol 51:41-44 
9. Dogra M, Singh R, Agarwal A et al (2017) Epidemiology of Uveitis in a Tertiary-care Referral Institute in North India. Ocul Immunol Inflamm 25:S46-S53

10. Sabhapandit S, Murthy SI, Singh VM et al (2017) Epidemiology and Clinical Features of Uveitis from Urban Populations in South India. Ocul Immunol Inflamm 25:S39-S45

11. Rahman Z, Ahsan Z, Rahman NA et al (2018) Pattern of Uveitis in a Referral Hospital in Bangladesh. Ocul Immunol Inflamm 26:893-896

12. Suzuki J, Oh-I K, Kezuka T et al (2010) Comparison of patients with ocular tuberculosis in the 1990 s and the 2000s. Jpn J Ophthalmol 54:19-23

13. Gupta A, Gupta V (2005) Tubercular posterior uveitis. Int Ophthalmol Clin 45:71-88

14. Bramante CT, Talbot EA, Rathinam SR et al (2007) Diagnosis of ocular tuberculosis: a role for new testing modalities? Int Ophthalmol Clin 47:45-62

15. Donahue HC (1967) Ophthalmologic experience in a tuberculosis sanatorium. Am J Ophthalmol 64:742-748

16. Biswas J, Badrinath SS (1995) Ocular morbidity in patients with active systemic tuberculosis. Int Ophthalmol 19:293-298

17. Lara LPR, Ocampo V (2013) Prevalence of presumed ocular tuberculosis among pulmonary tuberculosis patients in a tertiary hospital in the Philippines. J Ophthalmic Inflamm Infect 3:1-4

18. Beare NAV, Kublin JG, Lewis DK et al (2002) Ocular disease in patients with tuberculosis and HIV presenting with fever in Africa. $\mathrm{Br} \mathrm{J}$ Ophthalmol 86:1076-1079

19. Bouza E, Merino P, Muñoz $P$ et al (1997) Ocular tuberculosis. A prospective study in a general hospital. Med (Baltim) 76:53-61

20. Prevots DR, Marras TK (2015) Epidemiology of human pulmonary infection with nontuberculous mycobacteria: a review. Clin Chest Med 36:13-34

21. Stout JE, Koh W-J, Yew WW (2016) Update on pulmonary disease due to non-tuberculous mycobacteria. Int J Infect Dis IJID Off Publ Int Soc Infect Dis 45:123-134

22. Izumi K, Morimoto K, Hasegawa $\mathrm{N}$ et al (2019) Epidemiology of Adults and Children Treated for Nontuberculous Mycobacterial Pulmonary Disease in Japan. Ann Am Thorac Soc 16:341-347

23. Kheir WJ, Sheheitli H, Abdul Fattah M et al (2015) Nontuberculous Mycobacterial Ocular Infections: A Systematic Review of the Literature. Biomed Res Int 2015:164989

24. Jabs DA, Nussenblatt RB, Rosenbaum JT et al(2005) Standardization of uveitis nomenclature for reporting clinical data. Results of the First International Workshop. Am J Ophthalmol 140:509-16

25. Global tuberculosis report 2019 (2019) World Health Organization. https://www.who.int/tb/publications/global_report/en/ Accessed 1 March 2020

26. Rao NA, Saraswathy S, Smith RE (2006) Tuberculous uveitis: distribution of Mycobacterium tuberculosis in the retinal pigment epithelium. Arch Ophthalmol (Chicago, III 1960) 124:1777-9

27. Nazari H, Karakousis PC, Rao NA (2014) Replication of Mycobacterium tuberculosis in retinal pigment epithelium. JAMA Ophthalmol 132:724-729 
28. Goronzy JJ, Weyand CM (2005) T cell development and receptor diversity during aging. Curr Opin Immunol 17:468-475

29. Weng N-P (2006) Aging of the immune system: how much can the adaptive immune system adapt? Immunity 24:495-499

30. Akbar AN, Fletcher JM (2005) Memory T cell homeostasis and senescence during aging. Curr Opin Immunol 17:480-485

31. Ade S, Harries AD, Trébucq A et al (2014) National profile and treatment outcomes of patients with extrapulmonary tuberculosis in Bénin. PLoS One 9:e95603-e95603

32. Peto HM, Pratt RH, Harrington TA et al (2009) Epidemiology of extrapulmonary tuberculosis in the United States, 1993-2006. Clin Infect Dis 49:1350-1357

33. Borgdorff MW, Nagelkerke NJ, Dye C et al (2000) Gender and tuberculosis: a comparison of prevalence surveys with notification data to explore sex differences in case detection. Int $\mathrm{J}$ Tuberc Lung Dis 4:123-132

34. Martinez AN, Rhee JT, Small PM et al (2000) Sex differences in the epidemiology of tuberculosis in San Francisco. Int J Tuberc Lung Dis 4:26-31

\section{Tables}

Table 1. Clinical characteristics of patients with tuberculosis 


\begin{tabular}{|c|c|c|c|c|c|c|c|}
\hline & \multicolumn{2}{|c|}{ Total } & \multicolumn{2}{|c|}{ Ocular inflammation } & \multicolumn{2}{|c|}{ No ocular inflammation } & \multirow[t]{2}{*}{$P$ value } \\
\hline & No. & $\%$ & No. & $\%$ & No. & $\%$ & \\
\hline Number of patients & 101 & 100 & 7 & 100 & 94 & 100 & \\
\hline Age (years) & \multicolumn{2}{|l|}{$68.9 \pm 20.9$} & \multicolumn{2}{|l|}{$67.5 \pm 24.3$} & \multicolumn{2}{|l|}{$69.0 \pm 20.5$} & $0.50^{1}$ \\
\hline \multicolumn{8}{|l|}{$($ mean $\pm S D)$} \\
\hline Sex (Male : Female) & $58: 43$ & $57.4: 42.6$ & $1: 6$ & $14.3: 85.7$ & $57: 37$ & $60.6: 39.4$ & $0.01^{* 2}$ \\
\hline Foreign nationality & 8 & 7.9 & 1 & 14.3 & 7 & 7.4 & $0.55^{1}$ \\
\hline Extra pulmonary & 26 & 25.7 & 1 & 14.3 & 25 & 26.6 & $0.47^{1}$ \\
\hline \multicolumn{8}{|l|}{ tuberculosis } \\
\hline History of tuberculosis & 24 & 23.8 & 1 & 14.3 & 23 & 24.5 & $0.52^{1}$ \\
\hline Family history of & 12 & 11.9 & 2 & 28.6 & 10 & 10.6 & $0.21^{1}$ \\
\hline \multicolumn{8}{|l|}{ tuberculosis } \\
\hline Health care worker & 6 & 5.9 & 0 & 0 & 6 & 6.4 & $0.35^{1}$ \\
\hline History of autoimmune & 10 & 9.9 & 1 & 14.3 & 9 & 9.6 & $0.70^{1}$ \\
\hline \multicolumn{8}{|l|}{ disease } \\
\hline Immunosuppressive & 8 & 7.9 & 0 & 0 & 8 & 8.5 & $0.27^{1}$ \\
\hline \multicolumn{8}{|l|}{ therapy } \\
\hline History of cancer & 30 & 29.7 & 2 & 28.6 & 28 & 29.8 & $0.95^{1}$ \\
\hline History of & 2 & 2.0 & 1 & 14.3 & 1 & 1.1 & $0.02^{* 1}$ \\
\hline \multicolumn{8}{|l|}{ hematological disease } \\
\hline History of diabetes & 24 & 23.8 & 1 & 14.3 & 23 & 24.5 & $0.52^{1}$ \\
\hline History of uveitis & 0 & 0 & 0 & 0 & 0 & 0 & \\
\hline \multirow[t]{2}{*}{ Glaucoma } & $(16$ & $8.9(7.9)$ & 1 (2 eyes) & $14.3(14.3)$ & 8 (14 eyes) & $8.5(7.4)$ & $0.63^{1}$ \\
\hline & eyes) & & & & & & \\
\hline \multirow[t]{2}{*}{ Retinal disease } & $(23$ & $16.8(11.4)$ & 2 (3 eyes) & $28.6(21.4)$ & $(20$ & $16.0(10.6)$ & $0.38^{1}$ \\
\hline & eyes) & & & & eyes) & & \\
\hline History of ocular & 2 (2 eyes) & $2.0(1.0)$ & 0 & 0 & 2 (2 eyes) & $2.1(1.1)$ & $0.59^{1}$ \\
\hline trauma & & & & & & & \\
\hline
\end{tabular}

* : p<0.05, 1: t-test, 2: Pearson test 
Table 2. Clinical characteristics of 27 patients with NTM

\begin{tabular}{|c|c|c|}
\hline & \multicolumn{2}{|c|}{ Total } \\
\hline & No. & $\%$ \\
\hline Number of patients & 27 & 100 \\
\hline Age (years) (mean $\pm \mathrm{SD})$ & \multicolumn{2}{|l|}{$67.7 \pm 8.8$} \\
\hline Sex (Male : Female) & $8: 19$ & $29.6: 70.4$ \\
\hline Foreign nationality & 0 & 0 \\
\hline \multicolumn{3}{|l|}{ Species } \\
\hline Mycobacterium Intracellular & 13 & 48.1 \\
\hline Mycobacterium avium & 10 & 37.0 \\
\hline Mycobacterium Intracellular, Mycobacterium avium & 2 & 7.4 \\
\hline Mycobacterium Intracellular, Mycobacterium kansasii & 1 & 3.7 \\
\hline Mycobacterium Intracellular, Mycobacterium marseillense, Mycobacterium shimoidei & 1 & 3.7 \\
\hline History of tuberculosis & 2 & 7.4 \\
\hline Family history of tuberculosis & 1 & 3.7 \\
\hline Health care worker & 1 & 3.7 \\
\hline History of autoimmune disease & 3 & 11.1 \\
\hline Immunosuppressive therapy & 2 & 7.4 \\
\hline History of cancer & 2 & 7.4 \\
\hline History of hematological disease & 0 & 0 \\
\hline History of diabetes & 1 & 3.7 \\
\hline History of uveitis & 0 & 0 \\
\hline Glaucoma & 3 (6 eyes) & $11.1(11.1)$ \\
\hline Retinal disease & 3 (3 eyes) & $11.1(5.6)$ \\
\hline
\end{tabular}


Table 3. Characteristics of patients with tuberculosis who exhibited ocular inflammation

\begin{tabular}{|c|c|c|c|c|c|c|c|c|c|}
\hline Patient & Age(years) & Sex & Nationality & Laterality & Findings & $\begin{array}{c}\text { Avi } \\
\text { (OD/OS) }\end{array}$ & $\begin{array}{c}\text { Avf } \\
\text { (OD/OS) }\end{array}$ & $\begin{array}{l}\text { Duration between ATT } \\
\text { initiation and resolution of } \\
\text { ocular inflammation (days) }\end{array}$ & $\begin{array}{l}\text { Additional } \\
\text { treatment }\end{array}$ \\
\hline 1 & 68 & $\mathbf{F}$ & Japanese & os & $\begin{array}{l}\text { Anterior } \\
\text { inflammation }\end{array}$ & $1.0 / 1.0$ & $1.0 / 1.2$ & 29 & - \\
\hline 2 & 64 & $\mathbf{F}$ & Japanese & OU & Chorioretinitis & $1.0 / 0.9$ & Los & to follow-up & - \\
\hline 3 & 76 & F & Japanese & OU & $\begin{array}{l}\text { Retinal } \\
\text { vasculitis }\end{array}$ & $0.7 / 0.7$ & 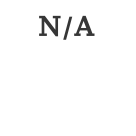 & 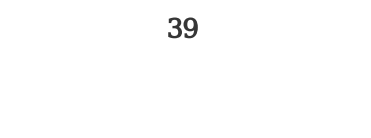 & - \\
\hline 4 & 40 & $\mathbf{F}$ & Japanese & os & $\begin{array}{l}\text { Snowball } \\
\text { opacities }\end{array}$ & $1.5 / 1.5$ & Los & to follow-up & - \\
\hline 5 & 96 & $\mathbf{F}$ & Japanese & OD & Chorioretinitis & $\begin{array}{l}0.03 / \\
0.03\end{array}$ & Los & to follow-up & - \\
\hline 6 & 19 & $\mathbf{M}$ & Filipino & OU & $\begin{array}{l}\text { Retinal } \\
\text { vasculitis }\end{array}$ & $1.2 / 1.5$ & $1.5 / 1.5$ & 77 & $\begin{array}{l}\text { Photocoagulation } \\
\text { to non-perfusion } \\
\text { area }\end{array}$ \\
\hline 7 & 83 & $\mathbf{F}$ & Japanese & OU & $\begin{array}{l}\text { Anterior } \\
\text { inflammation }\end{array}$ & $0.5 / 0.3$ & $0.4 / 0.6$ & 25 & $\begin{array}{c}\text { Topical } \\
\text { betamethasone }\end{array}$ \\
\hline
\end{tabular}

Avi; initial best-corrected visual acuity (BCVA), Avf; final BCVA, OS; left eye, OD; right eye, N/A; not available, OU; both eyes

Table 4. Multivariable analysis of risk factors for ocular inflammation in patients with tuberculosis 


\begin{tabular}{lcc}
\hline & Odds ratio & P value \\
\hline Sex (Female) & 10.5 & $0.04^{*}$ \\
\hline History of hematological disease & 22.7 & 0.08 \\
\hline
\end{tabular}

$*: \mathrm{p}<0.05$ 